\title{
Temporal Characteristics of the Predictive Synchronous Firing Modeled by Spike-Timing-Dependent Plasticity
}

\author{
Katsunori Kitano ${ }^{1}$ and Tomoki Fukai ${ }^{2,3,4}$ \\ ${ }^{1}$ Department of Computer Science, Ritsumeikan University, Kusatsu, Shiga 525-8577, Japan; ${ }^{2}$ Department of \\ Information-Communication Engineering, Tamagawa University, Machida, Tokyo 194-8610, Japan; ${ }^{3}$ Core Research for \\ Evolutional Science and Technology, Japan Science and Technology Corp., Kawaguchi, Saitama-ken, 332-0012, Japan
}

\begin{abstract}
When a sensory cue was repeatedly followed by a behavioral event with fixed delays, pairs of premotor and primary motor neurons showed significant increases of coincident spikes at times a monkey was expecting the event. These results provided evidence that neuronal firing synchrony has predictive power. To elucidate the underlying mechanism, here we argue some nontrivial characteristics of the predictive synchronous firing developed by spike-timing-dependent plasticity in a paradigm similar to classical conditioning. We find that the computationally developed synchrony shows the modulations of temporal precision, which are quite similar to those observed experimentally. Thus, our model suggests that the important characteristics of predictive synchronous firing, which were previously attributed to an animal's higher cognitive function, can emerge from a synaptic-level mechanism.
\end{abstract}

It is clearly advantageous for animals to detect and remember the causal relationships between events, to predict their occurrence and to prepare for a response (Barto et al. 1983; Desmond and Moore 1988: Tanji and Shima 1994; Schultz et al. 1997; Schultz 1998). Recent studies show that rate changes and synchronous firing of cortical neurons differentially engage in these cognitive functions. Riehle et al. (1997) trained monkeys on a delayed response task in which a GO signal to instruct a motor response repeatedly followed a sensory cue at several possible, fixed delays. Spikes from pairs of premotor and primary motor (PM/MI) neurons coincided more frequently than expected by chance, particularly at those times when the monkeys were expecting the GO signal. If the GO signal was actually presented at one of those times, the synchronous firing was followed by rate modulations. The statistically significant spike coincidence, independent of rate change, was termed "unitary event" (UE) and was considered to represent the monkey's anticipation of predictable events.

An interesting and nontrivial characteristic of UEs is that the degree of synchrony is strongly modulated in the time course of the individual trials: The longer the preparatory period for a motor response, the higher the temporal precision of spike coincidences between PM/MI neurons (Riehle et al. 2000). As suggested in the previous experimental study, this increase of temporal precision toward the end of each trial may be attributed to some higher cognitive processes in the monkey brain, such as update of temporal information. Here, we propose a different theoretical account for the temporal modulations, based on a synaptic-level mechanism.

It is widely accepted that the long-term plasticity of synapses underlies various changes in an animal's behavior, or learning. Synapses between cortical pyramidal neurons are strengthened or weakened if a presynaptic spike is followed or preceded by a postsynaptic spike, respectively (Markram et al. 1997; Bi and Poo 1998, 2001; Feldman 2000; Froemke and Dan 2002). The synapses modifiable by spike-timing-dependent plasticity (STDP)

\footnotetext{
${ }^{4}$ Corresponding author.

E-MAIL tfukai@eng.tamagawa.ac.jp; FAX 81 42-739-713.

Article and publication are at http://www.learnmem.org/cgi/doi/10.1101/ Im.64904.
}

compete for the timing of postsynaptic spikes and achieve several distinct functions, such as coincidence detection (Gerstner et al. 1996) and intersynaptic competition (Abbott and Nelson 2000; Song et al. 2000). Inputs that act in correlated groups can compete most successfully, implying coincidence detection. On the other hand, the same competition can establish the balanced excitation that keeps postsynaptic firing moderate and irregular (Song et al. 2000), implying activity regulation.

In this paper, we argue that STDP may provide a biologically plausible model of UEs. We show that the statistically significant number of spike coincidences occurs, with a reliable predictive power, only through the cooperation of the previously mentioned two STDP functions. Moreover, our simulations can account for the experimentally observed modulation pattern of the temporal precision of spike coincidences, without relying on any temporally nonhomogeneous neural process. Our model also predicts that the synchronous firing can be rapidly reorganized when the to-be-predicted times of events are changed. Although the two STDP functions are already known in the literature (Gerstner et al. 1996; Abbott and Nelson 2000; Song et al. 2000), our model demonstrates how they may cooperate in a specific cognitive function. Thus, this study shows an interesting example of the possible relationship between the synaptic plasticity and behavioral-level changes in neural responses, such as shown in the spatial navigation by the rat hippocampus (Mehta et al. 2002).

\section{RESULTS}

See Materials and Methods for details of our network model and simulations. Animals can predict events only if the brain knows how long it has been since a sensory cue, and this information must be accessible to the PM/MI neurons. Therefore, each PM/MI neuron in this model receives a sequence of timed spikes, besides background random spikes of constant rate and a brief depolarizing input representing a target event, that is, a GO signal (Fig. 1 ). In the timed spike sequence, some spikes (but not all) are initiated by and time-locked loosely to the sensory cue (Fig. 1), providing its temporal representation. Hereafter, the sequence may be termed a "loosely timed sequence" (LTS). The LTS plays a role similar to that of the so-called complete serial-compound 


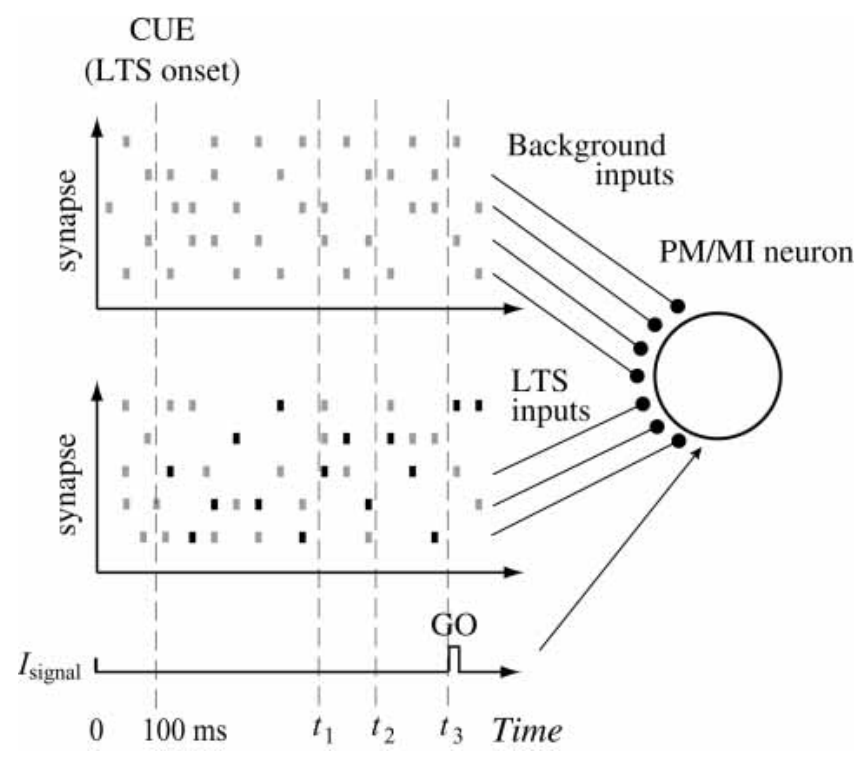

Figure 1 The cortical neural network model and simulated delayed response task. The neural network consists of 20 integrate-and-fire neurons that mimic monkey premotor or primary motor (PM/MI) neurons. Each neuron is innervated by a loosely timed firing sequence (LTS) consisting of 300 spike trains, 1300 Poisson-distributed spike trains that mimic noisy background inputs (1000 excitatory and 300 inhibitory inputs) from recurrent connections, and the input $\left(I_{\text {signal }}\right)$ representing the GO signal. The spike trains in the LTS, which differ for different neurons, consist of random spikes (gray bars) and spikes time-locked loosely to a sensory cue (black bars). The sensory cue initiates firing sequences of the loosely timed spikes at $100 \mathrm{msec}$; before the cue, each PM/MI neuron receives the random spikes in LTS and the background inputs. The timed spikes distribute uniformly through time and show, trial by trial, Gaussian-distributed timing jitters with a standard deviation of $10 \mathrm{msec}$. In this study, recurrent connections between the PM/MI neurons are not modeled explicitly. Thus, the effects of recurrent connections are represented only by the random background inputs in the present simulations. In a learning trial, the $\mathrm{GO}$ signal appears at one of the three predetermined times $\left(t_{1}, t_{2}\right.$, and $t_{3}=800,1300$, and $1800 \mathrm{msec}$, respectively) with equal probabilities. The GO signal is not presented in test trials.

stimulus in the conventional Hebbian learning theory of classical conditioning, that is, a spectrum of the timed processes initiated by a sensory cue (Barto et al. 1983; Montague et al. 1996; Moore et al. 1998). As the prefrontal cortex is generally considered to maintain timing information for organizing behaviors (Fuster 2001), the cortical region may be a candidate locus where we can find LTS. However, although there is much behavioral evidence that humans and animals can recognize intervals in the range of seconds (Matell and Meck 2000), the neural substrate has not yet been clarified. The existence of LTS must also be confirmed by further experiments.

The background random spikes may represent the continuous influences of recurrent feedback on the PM/MI neurons. In this study, different PM/MI neurons were innervated by completely different sets of background spikes and LTS spike trains. Therefore, no spurious spike coincidences will emerge from common modulations of input spikes.

All the excitatory synapses receiving the LTS or the background spikes are modifiable under the STDP rule found at the synapses between cortical pyramidal neurons. Namely, if a presynaptic spike at an excitatory synapse is followed by a postsynaptic spike, the peak conductance of the synapse is strengthened. For the reversed timing, the synapse is depressed. Parameter values were fixed such that the LTS and the background spikes coactivated the coincidence detection function (Gerstner et al. 1996) and the activity regulation function (Song et al. 2000) of
STDP on the individual PM/MI neurons. We show that the cooperation of the two functions is essential for the emergence of predictive synchronous firing of the PM/MI neurons from noisy background.

In the present study, the PM/MI neurons were trained similarly to the monkeys in the previous experiments (Riehle et al. 1997, 2000), as described below. In each learning trial, a cue signal appears at $100 \mathrm{msec}$ to initiate the timed spikes. Then, a GO signal appears with equal probabilities at one of three predetermined times (typically, $t_{1}=800, t_{2}=1300$, and $t_{3}=1800$ msec). According to experimental observations, the GO signal can always evoke spikes from each PM/MI neuron. In this study, the GO responses were induced in all the PM/MI neurons by a brief depolarizing input to these neurons. Possible delays of the GO responses in PM and MI from the visual stimulus (GO signal) were neglected. A learning trial is terminated $200 \mathrm{msec}$ after the occurrence of the GO signal. For the convenience of notation, we call the GO signal presented at the three times "GO1," "GO2," and "GO3," respectively.

One trial set of simulations consisted of 500 learning trials followed by 50 test trials. The spikes evoked in the test trials were used for the UE analysis explained later. During the learning and test trials, the mean temporal positions of the individual loosely timed spikes in each LTS were kept unchanged. In the monkey experiments, the GO signal was followed by the rate modulations. The GO signal, however, was not presented in the present test trials because we are only interested in the modulations of synchronous firing that occurs in the absence of the GO signal. The duration of a test trial was set to $2000 \mathrm{msec}$. A sufficient number of trial sets were performed to improve statistical power.

\section{The Self-Organizing LTS- and Background-Mediating Synapses}

Competition between synapses yielded bimodal weight distributions for both LTS- and background-mediating synapses and achieved the balanced excitation to regulate postsynaptic firing in a moderate range. Figure 2, A and B, display the weight distributions of the self-organizing LTS- and the backgroundmediating excitatory synapses, respectively. With an appropriately tuned activity regulation function, the distribution of LTSmediating synapses can be made more strongly polarized than that of the background-mediating synapses, as in the figures. In particular, the LTS-mediating synapses are dominant over the population of the most strengthened synapses (i.e., $g / g_{\max }>0.9$ ), which implies that postsynaptic spikes have developed stronger correlations with the loosely timed spikes than the background spikes.

To ascertain the dependence of the synaptic modifications on the individual spike times in an LTS, the LTS-mediating synapses and the corresponding LTS inputs were rearranged in descending order of synaptic weights (Fig. 2C). If a synapse was repeatedly activated shortly before some of GO1, GO2, or GO3 in the repetition of learning trials, the synapse tended to be the most strengthened. On the contrary, if a synapse was activated immediately after some GO signals, the synapse tended to be strongly depressed. Thus, even in the noisy background, the STDP rule preferentially enhanced those synapses consistently activated shortly before the GO signals by the loosely timed spikes that acted in correlated groups. We show later that the activity regulation function of STDP, which is implied by Figure 2 , A and B, is essential to the detection of those LTS spikes correlated with the GO responses.

\section{Coincidence Analysis}

Our analysis of spike coincidences follows the basic method proposed previously (Grün et al. 2002). For all 190 pairs of PM/MI 
Predictive Synchrony Modeled by STDP

A

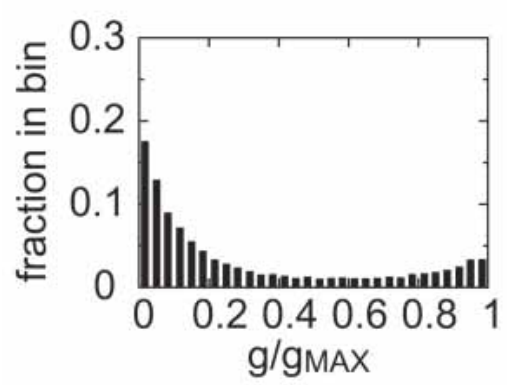

C
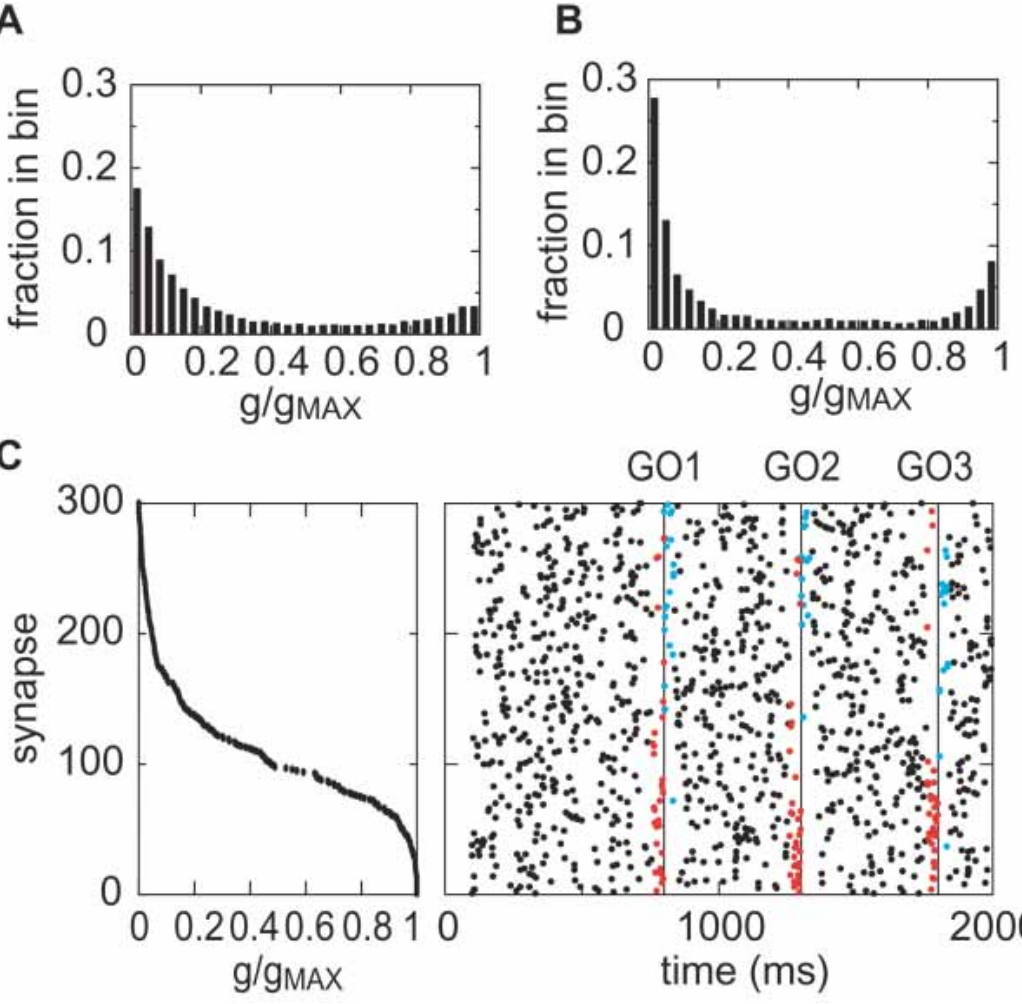

Figure 2 Competition between the self-organized synaptic weights. (A) Normalized weight distribution of background-mediating synapses. (B) Normalized weight distribution of LTS-mediating synapses. (C) The LTS-mediating synapses on a PM/MI neuron are rearranged in descending order of magnitudes (left), and the LTS spikes to the individual synapse are also shown in this same order (right). To see the relative times between the GO signals and the LTS spikes, those spikes that precede or follow the GO signals by $<40 \mathrm{msec}$ are represented in red or blue, respectively.

neurons, we calculated the rates of spike coincidences that occurred in a 100-msec-sliding time window in 5-msec steps. A pair of spikes can be regarded as coincident only if they are separated by less than this time step. Thus, the time step sets the measure for coincidence analysis and is hereafter called the "coincidence measure." We also calculated the accidental coincidence rate expected from the firing rates of each chosen pair based on the null hypothesis of independent firing. In each 100-msec time window, statistical significance of the excessive spike coincidence is calculated from a Poisson distribution (with the mean set to the expected number of coincidences) as the cumulative probability $P$ of observing the actual number of coincidences or even a larger one by chance. The larger the number of excessive coincidences, the closer $P$ is to 0 . Whenever $P$ is smaller than $5 \%$ in some 100 -msec time window, we regard it as an epoch with the statistically significant number of spike coincidences. We denote the occurrence time of the UE by the middle of this epoch.

Figure 3A displays the raster plots of a typical neuron pair in 50 test trials. In this figure, colored (blue and red) circles indicate coincident spikes; the red circles represent the coincident spikes in the epochs with the statistical significance of $P<0.05$. Figure $3 \mathrm{~B}$ shows the mean firing rates in each $100-$ msec time window. During the entire period of the trial, the rates were not significantly modulated. In Figure 3C, the time course of spike coincidences is shown in terms of the "joint-surprise" value defined as

$$
\log _{10}\left(\frac{1-P}{P}\right)
$$

which provides a better resolution in visualization than the raw
$P$-values. As in the previous experiment, the statistically significant number of the spike coincidences tended to occur in the neighborhood of the three anticipated times of the GO signal, even though it was not presented in the test trials. The raster plots of the neuron pair and the time course of spike coincidences are surprisingly similar to those obtained in the previous experiments.

In the UE analysis, 57 pairs show UEs at one of the expected times, 18 pairs at two of the expected times, and two pairs at all of the three expected times. Here, a UE was regarded as timelocked to an anticipated time, if the UE occurred within \pm 50 msec of that time. Whereas $\sim 10 \%$ of all neuron pairs showed no UEs, many pairs showed some UEs that were time-locked to none of the anticipated times. In the simulations, the number of the neuron pairs that fire coincidently at an anticipated time depends on the number of the LTS spike trains that happened to have the loosely timed spikes before that time. If we included a larger number of the LTS spike trains (and the corresponding synapses), the number of coincidently firing neuron pairs would be increased. Therefore, the present counts of neuron pairs should not be taken seriously. We only remark that not all the UEs were in fact time-locked to the GO signals in monkey motor cortices (Riehle et al. 1997, 2000).

Nevertheless, synchronous firing of sufficiently many PM/MI neurons could preserve the predictive power in the present simulations, as shown in the next section. The results indicate that spike synchrony provides a reliable prediction, if sufficiently many coincident spikes are detected for decoding.

\section{Dual Roles of STDP in the Predictive Synchrony}

The relatively high-frequency spontaneous firing of neurons in PM and MI was induced by a large number of the background inputs to the individual PM/MI neurons. If the rate of spontaneous firing is high, it would be difficult for these neurons to detect and maintain the correlations between postsynaptic spikes and the loosely timed spikes that are much less frequent than the background spikes in this model. Therefore, it would be difficult to develop UEs at a high rate of spontaneous firing.

Nevertheless, we can show that STDP maintains the statistical significance of spike coincidences against large changes in the activation level of the PM/MI neurons. To show this, we examined how the emergence of UEs depends on the average rate $r$ of the background activity, because it determines the firing rate of the PM/MI neurons. We conducted 10 sets of trials with different LTSs for each case of $r=10,20$, and 30 spikes/sec and performed the UE analysis for all the neuron pairs. Because of the activity regulation, the average output firing rate of the PM/MI neurons depended modestly on $r$ (Fig. 4A). If the activity regulation were absent, the rate would show a steeper, supralinear increase. Around the expected times of GO signal, the population firing rate showed a slight increase followed by weak depression, reflecting the temporal profile of the STDP learning window (Fig. $4 \mathrm{~B}$, bottom). Such modulations, however, were only visible in the mean firing rate averaged over all the PM/MI neurons (cf. Fig. 3B). In Figure 4B (upper three panels), the temporal distributions of epochs with the statistically significant number of spike coincidences were calculated in the following way: In each trial and each neuron pair, we counted the number of times that $P$ took a 
A

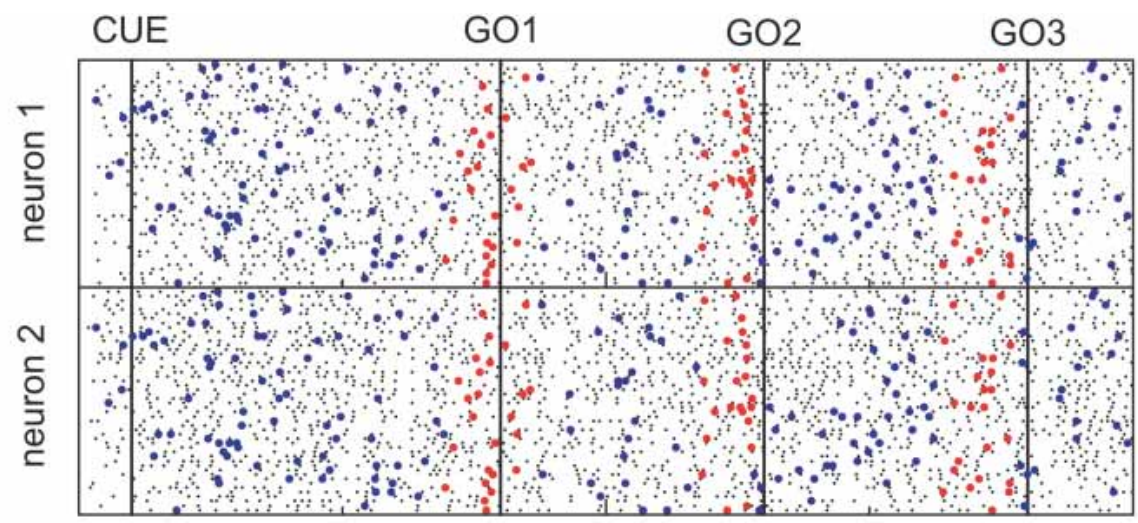

B

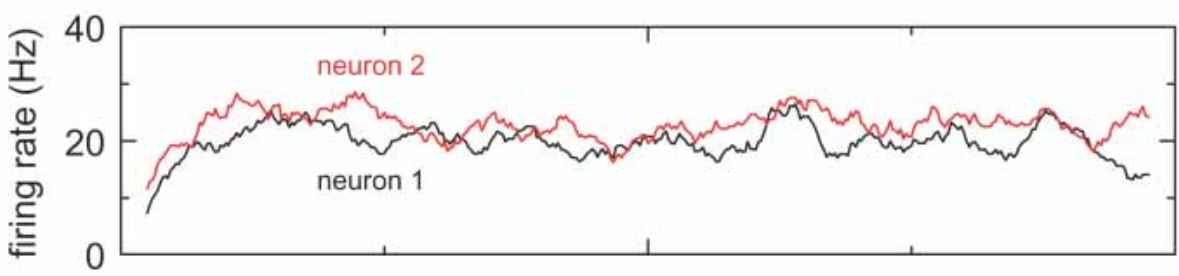

C

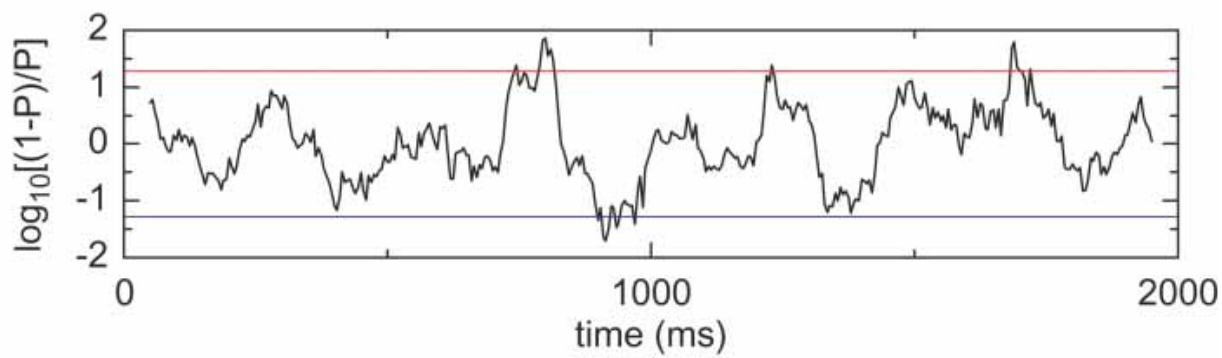

Figure 3 Raster plot of a typical PM/MI neuron pair and unitary events. $\sigma=10 \mathrm{msec}$ and $r=20 \mathrm{~Hz}$. (A) Spike raster obtained in 50 test trials for a typical neuron pair firing at an average rate of $20 \mathrm{spikes} / \mathrm{sec}$. Each tic or each colored dot represents one discharge. Blue dots indicate the coincident spikes that are statistically not significant. Red dots indicate UEs, namely, the coincident spikes that occur in the epochs with the statistically significant number of spike coincidences. (B) The time course of firing rates of the two neurons. The firing rate was evaluated in 100-msec windows in every 5-msec step. (C) The time course of spike coincidences is shown in terms of "joint-surprise" value: $\log _{10}[(1-P) / P]$. The value is calculated from the cumulative probability $P$ of observing the actual number of coincidences or even a larger one by chance in each 5 -msec bin and is plotted for the neuron pair shown above. If $P$ is $<5 \%$ (red line) in some bin, the number of spike coincidences in the corresponding 100-msec epoch (its middle is given by that bin) is regarded as statistically significant. Similarly, the epochs with significantly less excessive spike coincidences are defined by $P>0.95$ (blue line).

value $<5 \%$ in each time bin. Then, the count was summed up over all the neuron pairs and 10 trial sets for each value of $r$. We can see sharp peaks at the three anticipated times of the GO signal for relatively low background firing rates $(10$ and $20 \mathrm{~Hz}$ ). The peaks are less sharp, but still distinct for a background firing rate of $30 \mathrm{~Hz}$. Thus, the predictive coincident firing can be developed in a broad range of the background-firing rate.

To further confirm the essential role of the STDP-induced activity regulation in the emergence of the predictive synchronous firing, we enhanced the leading effect (Câteau and Fukai 2003) of synaptic potentiation by slightly increasing $A_{\mathrm{p}}$ from 0.01 to 0.0103 . This modification degrades activity regulation by less irregular neuronal firing; the coefficient of variation, $\mathrm{CV}=0.6$ for the new plasticity rule, whereas $\mathrm{CV}=0.9$ for the previous rule. Intuitively, we may expect that the enhanced longterm potentiation would improve the coincidence detection function of STDP and would make it easier for the PM/MI neurons to detect and "remember" the LTS spikes that coincide with the GO signal. However, this was not the case: UEs did not appear preferentially at the to-be-predicted times (Fig. 4C). The less effective activity regulation function prevented the neurons from establishing a balanced, irregular firing regime in which they are sensitive to the presynaptic spikes that act in correlated groups (Song et al. 2000). Such sensitivity is essential for selectively strengthening the synapses of the timed spikes correlated with the GO responses, while weakening the remaining synapses.

We also examined whether a multiplicative STDP rule is capable of organizing predictive synchronous firing: In the multiplicative rule, a synapse is strengthened $(\Delta t>0)$ or depressed $(\Delta t<0)$ by an amount $\Delta g=\left(g_{\max }-g\right) A_{\mathrm{p}} \exp \left(-\Delta t / \tau_{\mathrm{p}}\right)$ or $\Delta g=g A_{\mathrm{d}}$ $\exp \left(-|\Delta t| / \tau_{\mathrm{d}}\right)$, respectively. The rule induces no competition between synapses and consequently no activity regulation (Kistler and van Hemmen 2000; van Rossum et al. 2000; Rubin et al. 2001; Câteau and Fukai 2003). The results obtained (data not shown) were quite similar to those shown in Figure 4C; as expected, synchronous firing was not predictive in the absence of the activity regulation.

Finally, we examined whether a spike-timing-dependent learning rule, which operates similarly to the conventional ratebased Hebbian learning, may substitute for the (additive) STDP in predictive synchrony. In the modified learning rule, the LTD component was removed from the STDP learning rule. Thus, at every postsynaptic spike firing, both background-mediating and LTS-mediating synapses were strengthened according to the tem- 
A

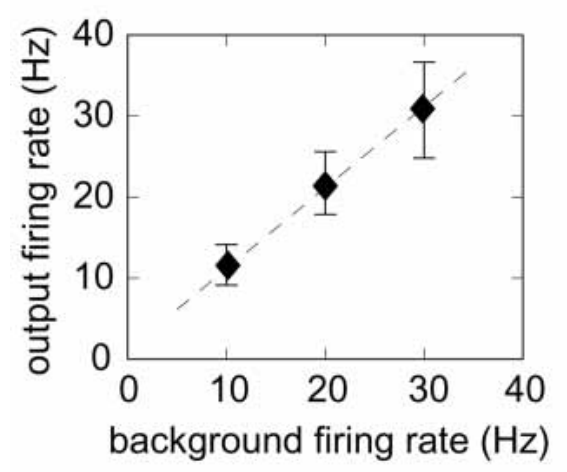

C

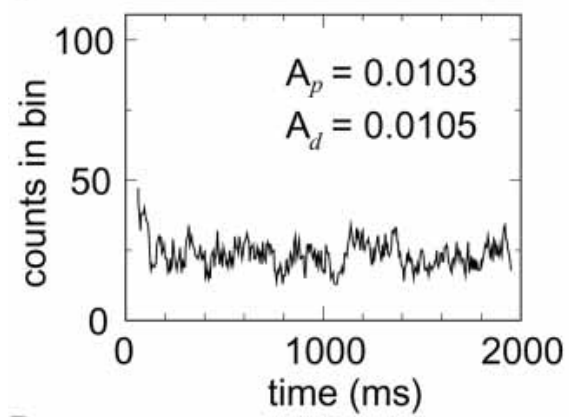

D

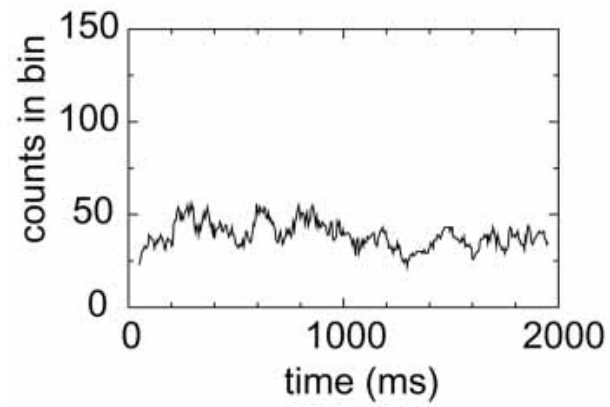

B

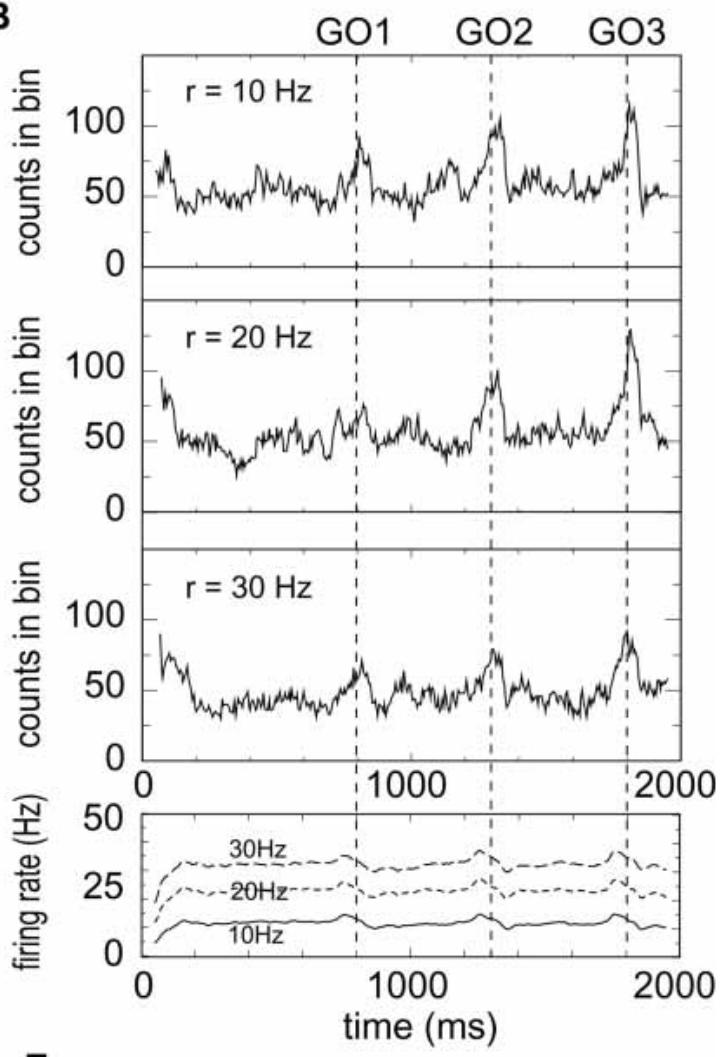

E

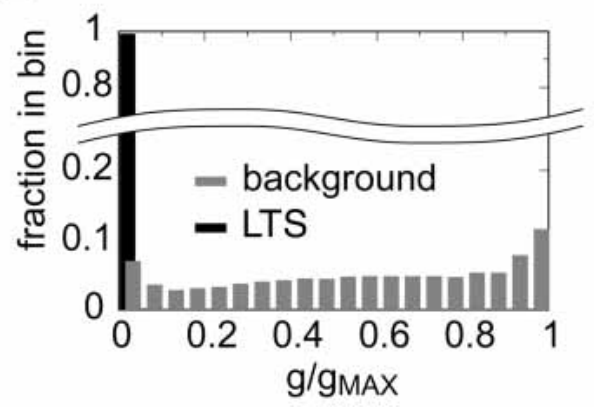

Figure 4 Detection of UEs at different rates $(r)$ of the excitatory background activity. ( $A$ ) Owing to the activity regulation function of STDP, the average firing rate of the individual PM/MI neurons vary only moderately against the modulations in $r$. Here, $\sigma=5 \mathrm{msec}$. (B) For $r=10 \mathrm{~Hz}, 20 \mathrm{~Hz}$, and $30 \mathrm{~Hz}$, we counted how many times the epoch with the statistically significant number of spike coincidences occurred in each time bin over all the PM/MI neuron pairs and 10 trial sets (upper three panels). The sharp peaks at the onset of trials in the cases of $r=20$ and $30 \mathrm{~Hz}$ were artifacts caused by the sudden raises in firing rate. The peaks are distinctly high at the expected times of the GO signal, especially at the time of GO3. The population firing rates averaged over all $20 \mathrm{PM} / \mathrm{Ml}$ neurons are shown for the different values of $r($ bottom). (C) The activity regulation function was made less effective by increasing the ratio $A_{\mathrm{p}} / A_{\mathrm{d}}$. In all of the present simulations with the increased ratio, the temporal distributions of the epochs with excessively many spike coincidences showed no predictive power. $(D)$ A similar distribution obtained for the Hebbian learning that induces a rate-based synaptic competition through a renormalization procedure. Coincident spikes exhibited no predictive power. $(E)$ The weight distribution of the self-organized synapses in the rate-based Hebbian learning.

poral delays $(\Delta t>0)$ from the latest presynaptic spikes at the individual synapses. Then, the synaptic weights were renormalized to keep the average of all the synaptic weights unchanged. Such a renormalization procedure has been known to induce a competition between synapses in the rate-based Hebbian learning (von der Marsburg 1973). Figure 4D shows the resultant UE distribution calculated as in Figure 4C. The temporal distribution exhibited no significant peaks predicting GO signals. The distribution of the self-organized excitatory synapses revealed that the winners were selected from the background-mediating synapses, whereas the LTS-mediating synapses were defeated in the competition (Fig. 4E). These results imply that in this learning rule, synapses compete for postsynaptic firing rate, and that in such a situation the LTS-mediating synapses stimulated at low spike rates have an extremely small chance to survive the competition.

\section{Changes in Temporal Precision of Spike Coincidences}

In the typical results of the experiments, the later the time of an excessive amount of spike coincidences, the higher the temporal precision of the spike coincidences. At late times (typically, $>500$ msec), the statistically significant number of spike coincidences could be detected even if the coincidence measure was as short as $2 \mathrm{msec}$, whereas excessively many spike coincidences were de- 
tectable at early times only with the coincidence measures greater than several milliseconds. It was speculated that the improvement of synchronicity at late times reflected a continuous update of temporal information through the formation of dynamical cell assemblies in the monkey brain (Riehle et al. 2000). However, there seems to be no a priori reason to expect that such an update preferably enhances, rather than suppresses, the spike coincidences toward the end of each trial.

The present model proposes a different mechanism for this interesting phenomenon. In Figure 5, we examined similar changes in the temporal precision of spike coincidences in the present model. We conducted the sliding time window analysis of the same data sets, varying the size of the coincidence mea- sure. We summed up the number of the times that the value of $P$ became $<5 \%$ in each time bin over all the neuron pairs and 10 trial sets. When the loosely timed spikes exhibited relatively large jitters in timing ( $\sigma=10 \mathrm{msec}$ ), the early peaks at $800 \mathrm{msec}$ were greatly suppressed compared with the peaks at $1800 \mathrm{msec}$ for all three values of the coincidence measure (Fig. 5A). Especially, the early peak almost disappeared for a 2-msec measure. When those timed spikes were precise $(\sigma=0 \mathrm{msec})$, the suppression of the early peaks was found only in the case of the 2-msec measure (Fig. 5B). Thus, the early spike coincidences are considered to occur in a broad temporal window of $5-10 \mathrm{msec}$, whereas the coincidences occur in a narrower time window at the late times. In the experiments, differences of a few hundred milliseconds in
A

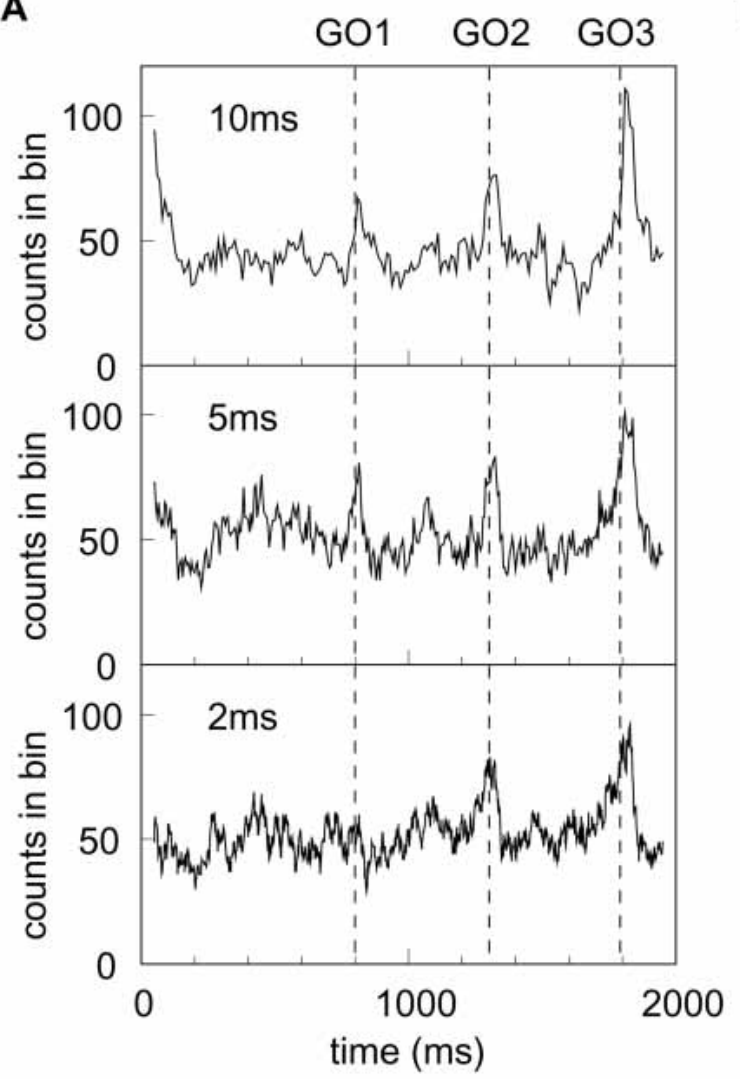

C

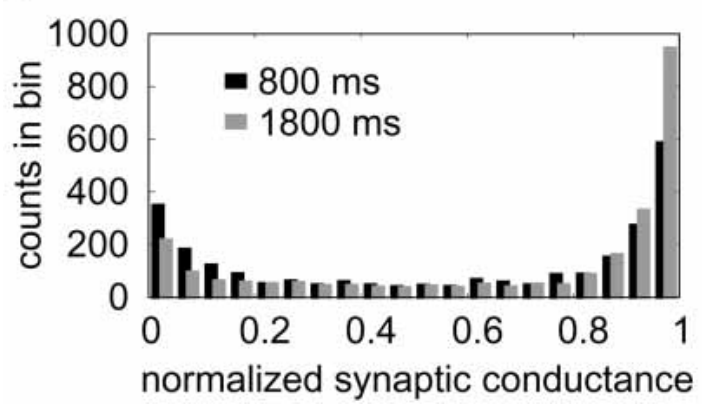

B

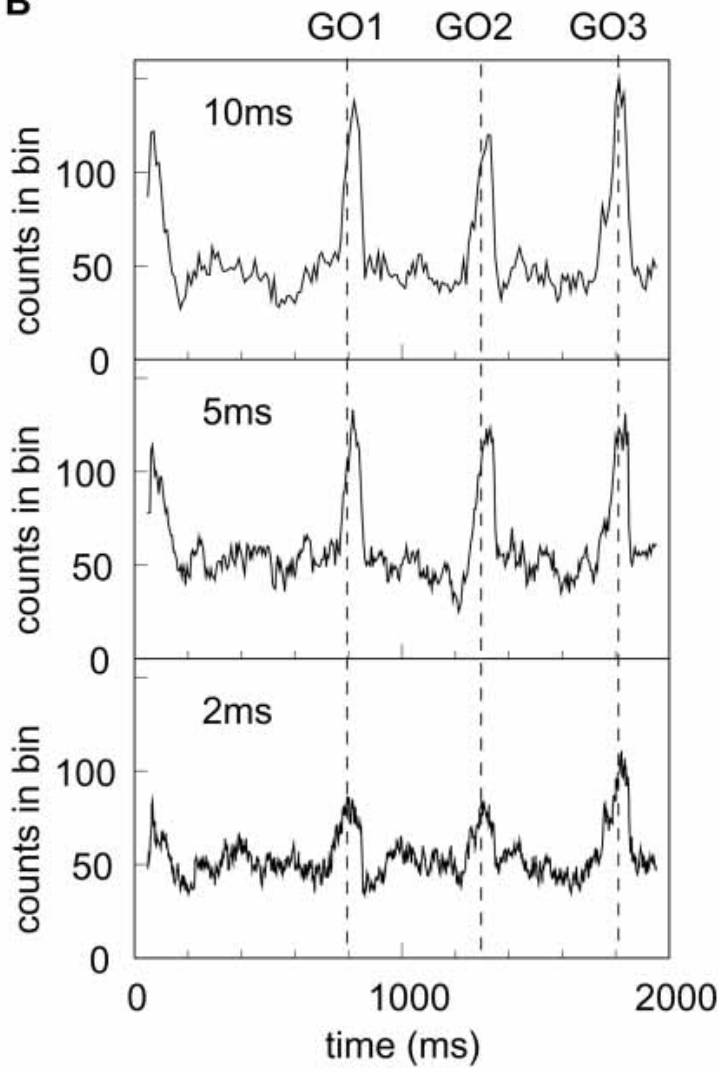

Figure 5 The modulations in the temporal precision of spike coincidences. The sliding time window analysis of the same data set was conducted with different coincidence measures $(10,5$, and $2 \mathrm{msec})$. Here, $r=10 \mathrm{~Hz}$. ( $A$ ) The later an anticipated time $(800,1300$, and $1800 \mathrm{msec})$, the higher the corresponding peak in the temporal distribution of the epochs with excessively many spike coincidences. The peak at 800 msec is invisible in the case of a 2 -msec window. The timing jitters were given by $\sigma=10 \mathrm{msec}$. (B) Similar temporal distributions were calculated in the case of $\sigma=0$ msec (precisely timed spikes). In this case, large suppression of early peaks can be seen only in the analysis with the 2-msec window. (C) Weight distributions of the LTS-mediating synapses responsible for the statistically significant number of spike coincidences at $t_{1}$ (black) and $t_{3}$ (gray) in the data set analyzed in $A$. 
the preparatory period resulted in large differences in the temporal precision of coincidences. Therefore, the model with loosely timed spikes agrees better with the experiments than that with precisely timed spikes.

The temporal precisions at different times are determined primarily by the conductance distributions of the LTS-mediating synapses responsible for the spike coincidences at $t_{1}, t_{2}$, and $t_{3}$ (we may term these synapses "GO1-synapses," "GO2-synapses," and "GO3-synapses," respectively). As shown in Figure 5C, the conductance distribution of the GO3-synapses is more strongly polarized toward the maximum than that of the GO1-synapses. This led to more precise spike coincidences at $t_{3}$ than at $t_{1}$. The mechanism underlying the differences in the conductance distributions can be understood as follows. In a learning trial, most synapses, except those strengthened by the GO response, undergo the depression caused by random inputs. The longer the trial, the stronger the depressing effects and the greater the consequent differences between the weakened and strengthened synapses. Because $t_{1}<t_{2}<t_{3}$, the GO1-synapses should suffer the strongest depressing effect, whereas the GO3-synapses suffer the weakest one. Therefore, the two synapse groups should be distributed differently, as shown in Figure 5C. Thus, our model accounts for the modulations of synchronicity based on the synaptic plasticity rule.

\section{The Role of the Random Spikes in LTS}

The random spikes in LTS spike trains are not correlated with the $\mathrm{GO}$ responses and hence cannot be associated with the responses by STDP. It is worthwhile studying the role of these spikes in the present simulations. Figure 6 shows the temporal distributions of the epochs with excessively many spike coincidences over all the neuron pairs in the absence of the random spikes. Similarly to Figures $4 \mathrm{~B}$ and $5, \mathrm{~A}$ and $\mathrm{B}$, the distributions were calculated for different values of the coincidence measure. For a 2-msec coincidence measure, the most prominent peak of the distribution appeared at the earliest time ( $800 \mathrm{msec})$. This temporal pattern is just opposite to those obtained in Figures $4 \mathrm{~B}$ and 5 , A and B, and to the experimental modulation pattern of the temporal precision of spike coincidences. In terms of the plasticity-based mechanism explained previously, the results can be understood as the effects of weakened noise.

\section{Reconstruction of New Predictions}

If the environment surrounding an animal changes, the animal must reconstruct a new model of the external world in its brain to behave appropriately. This relearning process should take place in the relevant neuronal circuits. It is often difficult to assess the relearning process in electrophysiological recording studies of behaving animals. It is still interesting, however, to examine whether and how the present neural network model modifies its organization to adapt to a new environment, which in the present context is defined as a new set of the times for the GO signal presentation.

The same network that was previously trained with the GO signals delivered at 800,1300 , and 1800 msec was retrained with a new set of signals delivered at 800,1100 , and 1800 msec. In Figure 7A, the temporal distributions of the epochs with excessively many spike coincidences are compared before and after 200 relearning trials. In the latter case, a sharp peak at $1300 \mathrm{msec}$ disappeared, while a new peak was appearing at $1100 \mathrm{msec}$. Figure $7 \mathrm{~B}$ displays the heights of the two peaks at $1300 \mathrm{msec}$ and $1100 \mathrm{msec}$ at various steps of the relearning process. As the relearning process proceeded, the peak heights at 1100 and 1300 msec gradually interchanged, which may represent a gradual exchange of their cognitive roles.

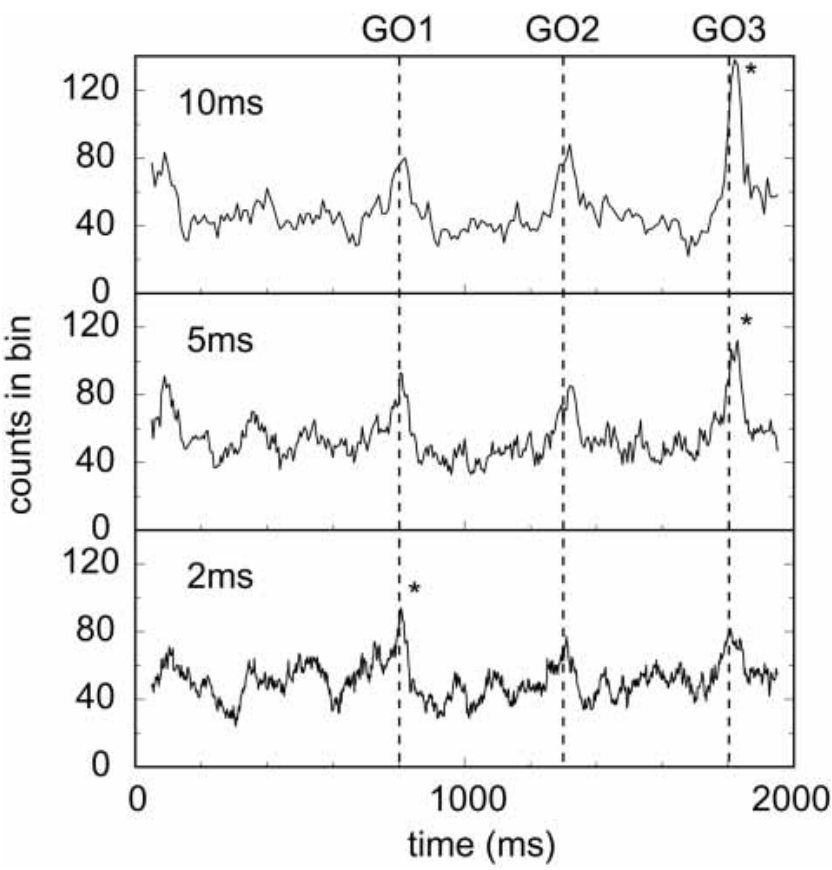

Figure 6 The temporal precision of spike coincidences in the absence of random spikes in LTS. The sliding time window analysis of the same data set was conducted with different coincidence measures $(2,5$, and 10 $\mathrm{msec}$ ), and the temporal distribution of the epochs with excessively many spike coincidences was calculated in each case. Here, $r=10 \mathrm{~Hz}, \sigma=10$ $\mathrm{msec}$, and the rate of time spikes in LTS was $2 \mathrm{~Hz}$. The highest peaks are designated by asterisks in the individual distributions. In experiments, the later an anticipated time of GO signal, the higher the temporal precision of spike coincidences. The simulation results shown here display different modulation patterns, which is evident from the distribution for the 2-msec coincidence measure (bottom).

\section{DISCUSSION}

Neuronal information coding may partly rely on spike timing or coherence (Ahissar et al. 2000; Steinmetz et al. 2000; Engel et al. 2001; Fries et al. 2001; Lu et al. 2001; Salinas and Sejnowski 2001; van Rullen and Thorpe 2001; Mehta et al. 2002). "Unitary events" were suggested to engage in representing an animal's expectancy of predictable events. In this study, we modeled UEs by means of the loosely timed firing sequences and STDP. STDP conditions PM/MI neurons to respond to the LTS spikes correlated with the GO responses of these neurons. We have demonstrated that the cooperation of the activity regulation function with the coincidence detection function is essential to the emergent predictive power of spike coincidences (Fig. 4C).

The biological reality of this model critically relies on the existence of LTS. It is noted that real LTS can be quite different from the set of LTS spike trains used in this study. For instance, each spike train of LTS may provide timed spikes only in a short temporal window, leaving the majority of the LTS spikes random. If a greater number of LTS spike trains are involved in the model, and if the brief packets of timed spikes in many LTS spike trains are distributed uniformly over time, we can easily duplicate essentially the same results as shown here.

Most interestingly, the present model achieves a novel insight into the mechanism underlying the changes in the temporal precision of spike coincidences (Fig. 5). It was argued that the phenomena reflected an internal update of temporal information in the monkey brain during the preparatory period. How this update, however, modulates the synchronous firing remained unclear. Alternatively, the improved synchronicity at 
A

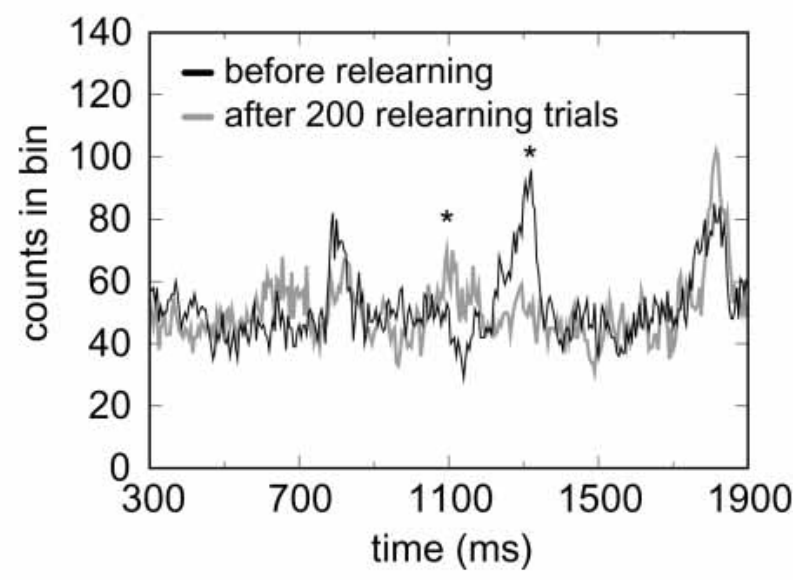

B

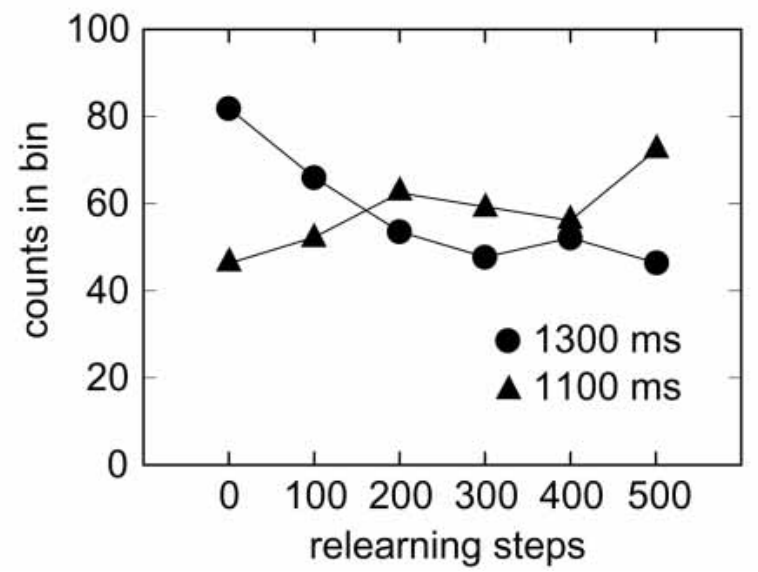

Figure 7 Relearning a new set of the $\mathrm{GO}$ signals delivered at $t_{1}=800$, $t_{2}=1100$, or $t_{3}=1800 \mathrm{msec}$ with equal probabilities. (A) Comparison between the temporal distributions of the epochs with excessively many spike coincidences before relearning and after 200 relearning trials. The asterisks at $1100 \mathrm{msec}$ and $1300 \mathrm{msec}$ show the two peaks of interest. (B) As the relearning process proceeded, the peak heights at 1100 and 1300 msec gradually interchanged until the former peak reached a maximal height and the latter peak diminished to a background level.

late times may reflect the animal's elevated attention or motivation (Steinmetz et al. 2000; Engel et al. 2001; Fries et al. 2001), as the conditional probability to have the GO signal was increased toward the end of each trial (e.g., if there are three equally likely times of the GO signal, the conditional probabilities to have it at the first, second, and third anticipated times are $1 / 3,1 / 2$, and 1 , respectively). This possibility, however, is also unlikely, because the epochs with excessively many spike coincidences also appeared at the times when animals were not expecting the GO signal. In the proposed model, the changes in synchronicity were induced during learning by the differences in the depressing effects on the individual synapses (Fig. 5C).

We have shown that the experimentally observed temporal modulations of the precision of spike coincidences can be much better understood in the presence, rather than absence, of the random spikes in LTS (Fig. 6). This, however, does not imply that these random spikes are useful for producing UEs that have predictive power. In fact, the peaks at the expected times of the GO signal were higher in Figure 6, where LTS had no random spikes, than in Figure 5A, where LTS had random spikes (in both simulations, $r=10 \mathrm{~Hz}$ and $\sigma=10 \mathrm{msec}$ ). These results simply suggest that the LTS spike trains, if they do exist and engage in the predictive synchrony, are likely to be noisy.

Another interesting feature predicted by this model is that the predictive spike coincidences reorganize flexibly to adapt to a new environment (Fig. 7). Because all excitatory synapses continuously undergo competition, whether they mediate LTSs or background activity, the changes in the GO responses easily reorganize the weight distribution of the synapses. The results imply that the learning performance does not heavily depend on the initial weight distribution. The initial distribution, however, may affect the temporal distribution of the spurious UEs that are not time-locked to the GO signals. If some timed spikes in a LTS spike train contribute to predictive synchronous firing at a certain time, other spikes in the same train have some chances to contribute to spurious UEs at different times. This explains why spurious UEs appear in this model, as was the case in experiments.

The standard multiplicative STDP rule (Kistler and van Hemmen 2000; van Rossum et al. 2000; Rubin et al. 2001) was incapable of organizing predictive synchrony. The results, however, do not exclude the possible emergence of predictive synchrony under multiplicative-type rules. According to the general theory of STDP (Câteau and Fukai 2003), it seems easy to modify the multiplicative rule so that it may show competition between synapses. Moreover, STDP has been studied in such a restricted experimental condition that only a limited number of synapses are activated on a postsynaptic cell. It has not been fully understood whether synapses undergo additional forms of competition, when a tremendous number of synapses are simultaneously activated. In fact, activity-dependent global scaling of synaptic weights (Turrigiano et al. 1998; Royer and Paré 2003) was suggested to induce an alternative form of competition (van Rossum et al. 2000). It was also shown recently that the plasticity rule depends on pairing pre- and postsynaptic spikes beyond the nearest neighbors (Froemke and Dan 2002). We have examined in the present model that such long-range interference within the individual spike trains does not remove competition between synapses, and hence should not change the qualitative results of this study.

The learning rule with the conventional synaptic normalization failed to give predictive power to synchronous firing in our model. This learning rule also yielded synaptic competition, selectively strengthening those synapses mediating presynaptic spikes of higher rates (Fig. 4E). Thus, the LTS-mediating synapses were strongly depressed as they received presynaptic spikes of low rate. On the other hand, the STDP learning (with an exponentially decaying learning time window and $A_{\mathrm{p}} \tau_{\mathrm{p}}<A_{\mathrm{d}} \tau_{\mathrm{d}}$ ) exerts stronger depressions on synapses at higher presynaptic firing rates. This characteristic of the STDP leads to an efficient postsynaptic activity regulation and improves the ability of neurons to detect the coincident spikes embedded in inputs of low spiking rates. Thus, both rate-based Hebbian learning and the STDP learning give rise to synaptic competition, but their functional roles are found to be quite different. It is noted that the virtue of STDP in the present model may disappear for strongly asymmetric STDP learning rules, as they may lead to the selective potentiation of higher-rate synaptic inputs (Burkitt et al. 2004).

In the present simulations, mutual connections between the PM/MI neurons were not explicitly included, and the effects of recurrent connections were replaced with a large amount of background spikes. Although the inclusion of mutual connections did not change the qualitative results of the present simulations (results not shown), to what extent the internal dynamical process among PM/MI neurons modulates the statistical significance 
of synchrony must be tested also in a large-scale network. Such simulations, unfortunately, require an enormous computational resource because the number of modifiable synapses becomes very large (here, 26,000 modifiable synapses were involved).

The expectancy-related responses of the basal ganglia (Montague et al. 1996; Schultz et al. 1997; Hollerman and Schultz 1998; Suri and Schultz 2001) and the cerebellum (Moore et al. 1998; Barto et al. 1999) have been successfully modeled using a temporal-difference (TD) learning algorithm (Barto et al. 1983) in reinforcement learning. Both the present model and these models represent temporal information by a cascade of the timetagged activities initiated by a sensory cue. We may model the time-tagged activities in terms of the so-called synfire network, that is, a layered network propagating a synchronous activity (Abeles 1991; Abeles et al. 1993; Prut et al. 1998; Diesmann et al. 1999; Câteau and Fukai 2001; Litvak et al. 2003). The synfire network propagates precisely timed spike sequence of 1-msec precision, for which the generation of UEs by STDP is obviously easier than for the less precise LTS (Kitano et al. 2003). As shown in Figure 5, however, sufficiently large modulations of the temporal precision of spike coincidences, which characterize the UEs, are found for such LTS that show 20- to 30-msec precision in spike timing. In fact, the rate changes with this degree of precision were found to be more informative than those spikes with 1-msec precision in the visual information processing (Oram et al. 1999; Richmond et al. 1999). Several ideas have been proposed for the neural representation of temporal information (Buonomano 2000; Matell and Mech 2000; Okamoto and Fukai 2001), but the issue is open for further experimental and theoretical studies.

\section{MATERIALS AND METHODS}

Here, we describe the mathematical details that are not given above. In the present study, we include $20 \mathrm{PM} / \mathrm{MI}$ neurons in the network shown in Figure 1. We model a PM/MI neuron using the leaky integrate-and-fire model described as

$$
\tau_{\mathrm{m}} \frac{d V}{d t}=E_{\text {leak }}-V+I_{\text {signal }}+I_{\text {Poisson }}+I_{\text {LTS }}
$$

with $\tau_{\mathrm{m}}=20 \mathrm{msec}, E_{\text {leak }}=-70 \mathrm{mV}$. When the membrane potential reaches a spike threshold of $-54 \mathrm{mV}$, the neuron generates an action potential and the membrane potential is reset to -60 $\mathrm{mV}$. The neurons are driven by three different input sources (Fig. 1). $I_{\text {signal }}$ is a depolarizing input representing a $\mathrm{GO}$ signal. At the GO signal, $I_{\text {signal }}$ is held at $8 \mathrm{mV}$ for $20 \mathrm{msec}$. The GO signal is assumed to always elicit spikes from the PM/MI neurons. $I_{\text {Poisson }}$ and $I_{\text {LTS }}$ represent the synaptic currents evoked by Poissondistributed spike trains and the loosely timed firing sequence, respectively. The background inputs are mediated by 1000 excitatory and 300 inhibitory synapses, and their typical mean rates are 10-20 spikes/sec at an excitatory synapse and 10 spikes/sec at an inhibitory synapse. Therefore, the present network model with 20 neurons involves 26,000 modifiable synapses. The background inputs induce relatively high-frequency spontaneous firing of $10-20 \mathrm{~Hz}$ in the individual PM/MI neurons, as observed in vivo.

We briefly mention possible finite size effects in the present simulations with 20 neurons. In fact, such effects seem to be only small. First, different neurons receive different LTS and background spikes. Therefore, no spurious spike coincidences are caused by common modulation by the external inputs. Second, the UE analysis does not count the spike coincidences expected from firing rates of neuron pairs (see Results). Thus, the analysis is tolerant of the rate fluctuations in a small neural population. Finally, and most importantly, we include all 190 neuron pairs in the analysis. This total number of neuron pairs is large enough for the statistical analysis.

An LTS consists of 300 spike trains that are mediated by 300 excitatory synapses. In our previous related study (Kitano et al.
2003; Kitano and Fukai 2004), we modeled the cascade of timetagged activities using the so-called synfire network, that is, a layered network propagating a synchronous activity (Abeles 1991). Synfire networks are known to produce spike sequence of even 1-msec precision (Diesmann et al. 1999; Câteau and Fukai 2001). In this study, we slightly relax the condition on the temporal precision of timed spikes. Each spike train of the LTS is a superposition of timed spikes and random spikes. The former spikes are initiated by and time-locked loosely to a sensory cue given at the beginning of every trial, are uniformly distributed over time at a rate of $2 \mathrm{spikes} / \mathrm{sec}$, and last until $200 \mathrm{msec}$ after appearance of the GO signal. These spikes show timing jitters from trial to trial around fixed times according to a Gaussian distribution of variance, $\sigma^{2}$. Typically, $\sigma=10 \mathrm{msec}$, but other values were also tested in several cases. The random spikes in the LTS obey a Poisson distribution with a mean rate of 3 spikes/sec.

A synaptic current is described as $I=g s\left(V-E_{\text {syn }}\right)$ with a gating variable $s$. If a presynaptic spike arrives at a synapse, $s$ is set as $s \rightarrow s+1.0$. Otherwise, $s$ decays exponentially with a 5 -msec decay constant. We set $E_{\mathrm{syn}}=E_{\mathrm{AMPA}}=0 \mathrm{mV}$ for excitatory synapses and $E_{\mathrm{syn}}=E_{\mathrm{GABA}}=-60 \mathrm{mV}$ for inhibitory synapses. The peak conductance $g$ is measured in the unit of the leak conductance. All excitatory synapses are modified according to the STDP rule given in the text. The relevant parameters are set as $A_{\mathrm{p}}=0.01$, $A_{\mathrm{d}}=0.0105, \tau_{\mathrm{p}}=\tau_{\mathrm{d}}=20 \mathrm{msec}$, and $g_{\max }=0.015$. The conductance of inhibitory synapses is fixed at $g_{\mathrm{GABA}}=0.05$.

The effects of STDP were simulated as follows. Suppose that a presynaptic spike at an excitatory synapse is followed by a postsynaptic spike at time $\Delta t$ later. Then, the peak conductance of the synapse is strengthened by an amount $\Delta g=g_{\max } A_{\mathrm{p}} \exp \left(-\Delta t / \tau_{\mathrm{p}}\right)$ if $\Delta t>0$, or depressed by an amount $\Delta g=g_{\max } A_{\mathrm{d}} \exp \left(-|\Delta t| / \tau_{\mathrm{d}}\right)$ if $\Delta t<0$. The width of the learning time window is set as $\tau_{\mathrm{p}}=\tau_{\mathrm{d}}=20 \mathrm{msec}$. The parameters $A_{\mathrm{p}}$ and $A_{\mathrm{d}}$ are determined such that the leading depression effect $A_{\mathrm{d}} \tau_{\mathrm{d}}$ is $5 \%$ larger than the leading potentiation effect $A_{\mathrm{p}} \tau_{\mathrm{p}}$. In most simulations, the LTSmediating synapses were initially distributed according to a Gaussian distribution with mean $\approx 0.5 g_{\max }$ and standard deviation $\approx 0.2 g_{\max }$. The background-mediating synapses were initially set at the maximal conductance. However, the learning performance of the present model was almost independent of initial conditions.

\section{ACKNOWLEDGMENTS}

We thank H. Câteau for helpful discussions. One of the present authors (K.K.) was supported by the Japan Society for Promotion of Science (JSPS).

The publication costs of this article were defrayed in part by payment of page charges. This article must therefore be hereby marked "advertisement" in accordance with 18 USC section 1734 solely to indicate this fact.

\section{REFERENCES}

Abbott, L.F. and Nelson, S.B. 2000. Synaptic plasticity: Taming the beast. Nat. Neurosci. 3 Suppl: 1178-1183.

Abeles, M. 1991. Corticonics. Cambridge Univ. Press, Cambridge, UK.

Abeles, M., Bergmann, H., Margalit, E., and Vaadia, E. 1993. Spatiotemporal firing patterns in the frontal cortex of behaving monkeys. J. Neurophysiol. 70: 1629-1638.

Ahissar, E., Sosnik, R., and Haidarliu, S. 2000. Transformation from temporal to rate coding in a somatosensory thalamocortical pathway. Nature 406: 302-306.

Barto, A.G., Sutton, R.S., and Anderson, C.W. 1983. Neuron-like adaptive elements that can solve difficult learning control problems. IEEE Trans. Sys. Man Cyber. 15: 835-846.

Barto, A.G., Fagg, A.H., Sitkoff, N., and Houk, J.C. 1999. A cerebellar model of timing and prediction in the control of reaching. Neural. Comput. 11: 565-594.

Bi, G.-Q. and Poo, M.-M. 1998. Activity-induced synaptic modifications in hippocampal culture: Dependence on spike timing, synaptic strength and cell type. J. Neurosci. 18: 10464-10472. . 2001. Synaptic modification by correlated activity: Hebb's postulate revisited. Annu. Rev. Neurosci. 24: 139-166.

Buonomano, D.V. 2000. Decoding temporal information: A model based on short-term synaptic plasticity. J. Neurosci. 20: 1129-1141. 
Burkitt, A.N., Meffin, H., and Grayden, D.B. 2004. Spike-timing-dependent plasticity: The relationship to rate-based learning for models with weight dynamics determined by a stable-fixed point. Neural Comput. 16: 885-940.

Câteau, H. and Fukai, T. 2001. Fokker-Planck approach to the pulse packet propagation in synfire chain. Neural Netw. 14: 675-685. . 2003. A stochastic method to predict the consequence of arbitrary forms of spike-timing-dependent plasticity. Neural Comput. 15: $597-620$.

Desmond, J.E. and Moore, J.W. 1988. Adaptive timing in neural networks: The conditioned response. Biol. Cybern. 58: 405-415.

Diesmann, D., Gewaltig, M.O., and Aertsen, A. 1999. Stable propagation of synchronous spiking in cortical neural networks. Nature 402: 529-533.

Engel, A.K., Fries, P., and Singer, W. 2001. Dynamic predictions: Oscillations and synchrony in top-down processing. Nat. Rev. Neurosci. 2: 704-716.

Feldman, D.E. 2000. Timing-based LTP and LTD at vertical inputs to layer II/III pyramidal cells in rat barrel cortex. Neuron 27: 45-56.

Fries, P., Reynolds, J.H., Rorie, A.E., and Desimone, R. 2001. Modulation of oscillatory neuronal synchronization by selective visual attention. Science 291: 1560-1563.

Froemke, R.C. and Dan, Y. 2002. Spike-timing-dependent synaptic modification induced by natural spike trains. Nature 416: $433-438$.

Fuster, J.M. 2001. The prefrontal cortex-An update: Time is of the essence. Neuron 30: 319-333.

Gerstner, W., Kempter, R., van Hemmen, J.L., and Wagner, H. 1996. A neuronal learning rule for sub-millisecond temporal coding. Nature 383: 76-78.

Grün, S., Diesmann, M., and Aertsen, A. 2002. Unitary events in multiple single-neuron spiking activity: I. Detection and significance, II. Nonstationary data. Neural Comput. 14: 43-119.

Hollerman, J.R. and Schultz, W. 1998. Dopamine neurons report an error in the temporal prediction of reward during learning. Nat. Neurosci. 1: 304-309.

Kistler, W.M. and van Hemmen, J.L. 2000. Modeling synaptic plasticity in conjunction with the timing of pre- and postsynaptic action potentials. Neural Comput. 12: 385-405.

Kitano, K. and Fukai, T. 2004. Predictive synchrony organized by spike-based Hebbian learning with time-representing synfire activities. In Neural information processing: Research and development (eds. L. Wang and J.C. Rajapakse). Springer-Verlag, Singapore (in press).

Kitano, K., Okamoto, H., and Fukai, T. 2003. Time representing cortical activities: Two models inspired by prefrontal persistent activity. Biol. Cybern. 88: 387-394.

Litvak, V., Sompolinsky, H., Segev, I., and Abeles, M. 2003. On the transmission of rate code in long feedforward networks with excitatory-inhibitory balance. J. Neurosci. 23: 3006-3015.

Lu, T., Liang, L., and Wang, X. 2001. Temporal and rate representations of time-varying signals in the auditory cortex of awake primates. Nat. Neurosci. 4: 1131-1138.

Markram, H., Lubke, J., Frotscher, M., and Sakmann, B. 1997. Regulation of synaptic efficacy by coincidence of postsynaptic APs and EPSPs. Science 275: 213-215.

Matell, M.S. and Meck, W.H. 2000. Neuropsychological mechanisms of interval timing behavior. BioEssays 22: 94-103.

Mehta, M.R., Lee, A.K., and Wilson M.A. 2002. Role of experience and oscillations in transforming a rate code into a temporal code. Nature 417: 741-746.
Montague, P.R., Dayan, P., and Sejnowski, T.J. 1996. A framework for mesencephalic dopamine systems based on predictive Hebbian learning. J. Neurosci. 16: 1936-1947.

Moore, J.W., Choi, J.-S., and Brunzell, D.H. 1998. Neural, psychological and computational perspectives. In Timing of behavior (eds. D.A. Rosenbaum and C.E. Collyer), pp. 3-34. MIT Press, Cambridge, MA.

Okamoto, H. and Fukai, T. 2001. Neural mechanism for a cognitive timer. Phys. Rev. Lett. 86: 3919-3922.

Oram, M.W., Wiener, M.C., Lestienne, R., and Richmond, B.J. 1999. Stochastic nature of precisely timed spike patterns in visual system neural responses. J. Neurophysiol. 81: 3021-3033.

Prut, Y., Vaadia, E., Bergman, H., Haalman, I., Slovin, H., and Abeles, M. 1998. Spatiotemporal structure of cortical activity: Properties and behavioral relevance. J. Neurophysiol. 79: 2857-2874.

Richmond, B.J., Oram, M.W., and Wiener, M.C. 1999. Response features determining spike times. Neural Plast. 6: 133-145.

Riehle, A., Grün, S., Diesmann, M., and Aertsen, A. 1997. Spike synchronization and rate modulation differentially involved in motor cortical function. Science 278: 1950-1953.

Riehle, A., Grammont, F., Diesmann, M., and Grün, S. 2000. Dynamical changes and temporal precision of synchronized spiking activity in monkey motor cortex during movement preparation. J. Physiol. Paris 94: 569-582.

Royer S. and Paré, D. 2003. Conservation of total synaptic weight through balanced synaptic depression and potentiation. Nature 422: 518-522.

Rubin, J., Lee, D.D., and Sompolinsky, H. 2001. Equilibrium properties of temporally asymmetric Hebbian plasticity. Phys. Rev. Lett. 86: $364-367$.

Salinas, E. and Sejnowski, T.J. 2001. Correlated neuronal activity and the flow of neural information. Nat. Rev. Neurosci. 2: 539-550.

Schultz, W. 1998. Predictive reward signal of dopamine neurons. J. Neurophysiol. 80: 1-27.

Schultz, W., Dayan, P., and Montague, P.R. 1997. A neural substrate of prediction and reward. Science 275: 1593-1599.

Song, S., Miller, K.D., and Abbott, L.F. 2000. Competitive Hebbian learning through spike-timing-dependent synaptic plasticity. Nat. Neurosci. 3: 919-926.

Steinmetz, P.N., Roy, A., Fitzgerald, P.J., Hsiao, S.S., Johnson, K.O., and Niebur, E. 2000. Attention modulates synchronized neuronal firing in primate somatosensory cortex. Nature 404: 187-190.

Suri, R.E. and Schultz, W. 2001. Temporal difference model reproduces anticipatory neural activity. Neural Comput. 13: 841-862.

Tanji, J. and Shima, K. 1994. Role of supplementary motor area cells in planning several movements ahead. Nature 371: 413-416.

Turrigiano, G.G., Leslie K.R., Desai, N.S., Rutherford, L.C., and Nelson, S.B. 1998. Activity-dependent scaling of quantal amplitude in neocortical neurons. Nature 391: 892-896.

van Rossum, M.C., Bi, G.Q., and Turrigiano, G.G. 2000. Stable Hebbian learning from spike timing-dependent plasticity. J. Neurosci. 20: $8812-8821$.

van Rullen, R. and Thorpe, S.J. 2001. Rate coding versus temporal order coding: What the retinal ganglion cells tell the visual cortex. Neural Comput. 13: $1255-1283$.

von der Marsburg, C. 1973. Self-organization of orientation sensitive cells in the striate cortex. Kybernetik 14: 85-100.

Received June 18, 2003; accepted in revised form February 24, 2004. 


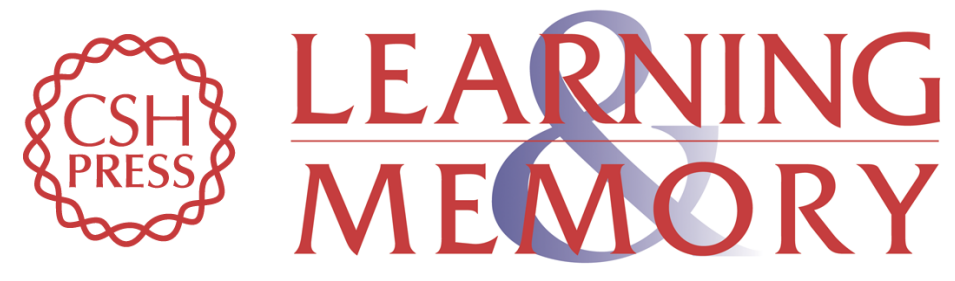

\section{Temporal Characteristics of the Predictive Synchronous Firing Modeled by Spike-Timing-Dependent Plasticity}

Katsunori Kitano and Tomoki Fukai

Learn. Mem. 2004, 11:

Access the most recent version at doi:10.1101/lm.64904

References This article cites 47 articles, 9 of which can be accessed free at: http://learnmem.cshlp.org/content/11/3/267.full.html\#ref-list-1

License

Email Alerting Receive free email alerts when new articles cite this article - sign up in the box at the Service top right corner of the article or click here. 